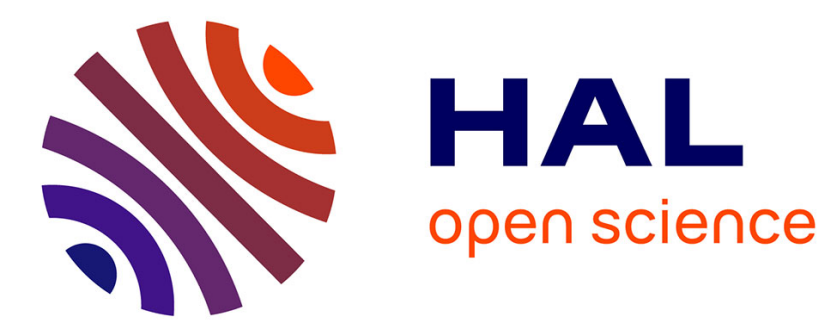

\title{
Le Ciane, un collectif hybride dans le monde de la périnatalité
}

\author{
Madeleine Akrich
}

\section{To cite this version:}

Madeleine Akrich. Le Ciane, un collectif hybride dans le monde de la périnatalité. Revue de médecine périnatale, 2010, 2, pp.197-202. 10.1007/s12611-010-0083-7 . hal-00549797

\section{HAL Id: hal-00549797}

https://hal-mines-paristech.archives-ouvertes.fr/hal-00549797

Submitted on 18 Apr 2012

HAL is a multi-disciplinary open access archive for the deposit and dissemination of scientific research documents, whether they are published or not. The documents may come from teaching and research institutions in France or abroad, or from public or private research centers.
L'archive ouverte pluridisciplinaire HAL, est destinée au dépôt et à la diffusion de documents scientifiques de niveau recherche, publiés ou non, émanant des établissements d'enseignement et de recherche français ou étrangers, des laboratoires publics ou privés. 


\title{
Le Ciane, un collectif hybride dans le monde de la périnatalité
}

\author{
The Ciane, a hybrid collective within the perinatality domain
}

\author{
M. Akrich \\ Reçu le 12 août 2010 ; accepté le 17 septembre 2010 \\ (C) Springer-Verlag France 2010
}

Résumé Le collectif interassociatif autour de la naissance (Ciane) regroupe des associations dont les histoires, les ressources et les compétences sont variées ; grâce aux outils de coordination fournis par Internet, il a pu construire une articulation entre une expertise tirée de l'expérience des femmes et des couples et une expertise médicoscientifique. De cette manière, il a tiré partie de la diversité de ses membres pour constituer un mouvement original entièrement tourné vers l'action politique au sens large. Pour citer cette revue : Rev. Méd. Périnat. 2 (2010).

\section{Mots clés Usagers · Association · Politique · Expertise profane}

\begin{abstract}
The "collectif interassociatif autour de la naissance, Ciane" brings together organisations with various backgrounds, resources and competencies; thanks to Internet coordination tools, it succeeded in articulating an expertise gained from experience of women and couples with a medico-scientific expertise. Thus, it took advantage of the diversity of its members to constitute a movement oriented towards policy action at large. To cite this journal: Rev. Méd. Périnat. 2 (2010).
\end{abstract}

\section{Keywords Consumers · Association · Policy · Lay} expertise

Le collectif interassociatif autour de la naissance (Ciane) a été constitué en 2003 et s'est rapidement imposé comme l'acteur principal représentant au niveau national les usagers

M. Akrich $(\square)$

Centre de sociologie de l'innovation, mines ParisTech, 60, boulevard Saint-Michel, F-75006 Paris, France e-mail : madeleine.akrich@mines-paristech.fr

Madeleine Akrich est sociologue et dirige le centre de sociologie de l'innovation. Elle est membre du CA du Ciane. dans le domaine de la périnatalité ${ }^{1}$. D'autres associations avaient eu une ambition du même ordre par le passé, sans toutefois arriver à la concrétiser. Le contexte créé par la loi du 4 mars 2002, qui instituait une représentation des usagers dans tous les organismes, conseils, etc. ayant à traiter des questions de santé, a joué un rôle décisif en installant l'espace dans lequel le Ciane pouvait se développer. Au-delà de cet aspect conjoncturel, parce qu'il a regroupé sur une base ouverte tous les groupes d'usagers qui s'étaient investis sur ces questions, le Ciane a pu ainsi mettre à profit la diversité des expériences et des compétences présentes en leur sein. Dans cet article, nous nous proposons de décrire ce dont le Ciane est constitué ainsi que la manière dont il fonctionne afin d'apporter quelques éléments de réponse aux interrogations légitimes des professionnels face à ce nouvel acteur.

\section{Ciane, un mouvement composite}

Le Ciane est un regroupement d'associations et ne comporte pas de membres individuels. Les associations qui le composent sont diverses et ont été créées avec des motivations de départ variées :

- dans un certain nombre de cas, l'association s'est constituée à partir d'un événement déclencheur : menace de fermeture d'un établissement, attaques engagées contre un professionnel atypique, etc. Lorsque la cause initiale du regroupement disparaît, s'ensuit une période de « reconversion », qui peut s'accompagner d'une baisse des effectifs, pendant laquelle la communauté créée autour de l'événement essaie de se maintenir, de se définir une identité, des buts collectifs, tournés vers elle-même et/ou tournés vers l'extérieur (extension de la « cause », volonté d'influer sur l'organisation de la périnatalité);

- une part importante d'associations anciennes est née autour d'une communauté d'expérience, souvent un

\footnotetext{
${ }^{1}$ Pour une description plus détaillée de la création du Ciane et de son inscription dans l'histoire des mouvements français autour de la périnatalité, voir Phan [1].
} 


\section{Historique rapide des mouvements nés d'Internet}

La liste Naissance a été créée en 1999 afin de permettre « un échange d'informations, de points de vue, de réflexions, de vécus... autour de la naissance et du parentage. » Bien que la liste fonctionne sur le principe d'un engagement des personnes en leur nom propre et sur la base de leur expérience personnelle, les fondateurs de la liste — un petit groupe d' « usagers » et quelques professionnels - sont au fond motivés par l'envie que les choses changent dans le domaine de la périnatalité, même s'ils ne sont pas prêts à recourir aux formes classiques de la mobilisation collective.

Assez vite, un site web est créé, qui met à disposition l'ensemble des « ressources » construites par la liste : bibliographie, documents, discussions, récits de naissances, coordonnées d'associations.

En mai 2001, deux ans environ après le démarrage de la liste et quelques mois après la création du site, une des personnes fondatrices décide de lancer une newsletter publique par mail, destinée à diffuser des informations sur « des conférences, nouveaux sites, dossiers édités, livres, activités associatives et témoignages ». Cette lettre diffuse en moyenne une quinzaine d'informations par mois et est envoyée à près de 1200 abonnés.

À la même époque, une abonnée à la liste Naissance constitue une liste de discussion sur la césarienne pour « permettre aux femmes ayant subi une ou plusieurs césariennes de témoigner, de parler de

groupe de parents qui ont choisi d'accoucher à domicile ou d'avoir recours à un accompagnement particulier. Il s'agit à la fois de prolonger cette expérience partagée et d'en préserver/développer la possibilité pour de nouveaux parents ;

- d'autres associations, en plus petit nombre, ont été créées à partir d'une expérience personnelle et d'une volonté de partage, d'aide : c'est le cas d'associations autour de l'allaitement ou de la parentalité. À l'exception de la Leche League $^{2}$, organisation nationale et même internationale, ces associations sont souvent centrées au départ sur une personne ou un petit groupe qui « offre » quelque chose. Plus récemment, d'autres associations d'envergure nationale ont vu le jour autour de problèmes spécifiques comme la césarienne (Césarine) ou la difficulté maternelle (Maman Blues) : bien que très soucieuses d'apporter une aide aux personnes confrontées au problème dont elles traitent, elles se sont aussi mobilisées rapidement sur le terrain politique de la transformation des pratiques et de l'organisation des soins, ce qui les rapproche du quatrième type que nous avons distingué ;

- ces dernières associations ont émergé d'emblée autour d'une volonté de transformation large des pratiques de la

\footnotetext{
${ }^{2}$ Sur l'histoire de la Leche League, on peut voir les articles de Weiner [2] et de Sandre-Pereira [3].
}

leur vécu, de s'exprimer au milieu d'autres qui comprennent »; en 2005, une association, Césarine, est créée dans le prolongement de la liste de discussion.

La rédactrice de la Newsletter met sur pied, en février 2003, suite à un long débat autour de ce thème sur la liste Naissance, une liste de discussion sur les maisons de naissance - dont la mise en place en France est bloquée par l'opposition des médecins et de l'administration de la santé - , puis dans la foulée une autre liste à archives publiques, Re-Co-Naissances, ouverte à tous ceux qui souhaitent « discuter des possibilités, projets, envisager des actions pour " faire bouger" les choses dans le domaine de la Naissance ». En mai 2003, elle propose d'instaurer une semaine mondiale de l'accouchement respecté (Smar) et de créer une association autour de cet objectif, l'Alliance francophone pour l'accouchement respecté (Afar). En avril 2004, une colistière lance une nouvelle liste de soutien sur l'épisiotomie, qui débouche un an et demi après sur la création d'un site d'information spécialisé.

Notons que la liste Naissance a été fermée en 2007 par son fondateur qui a estimé que les discussions, de plus en plus monopolisées par des personnes ayant fait des choix de vie très marqués (de l'accouchement à domicile jusqu'à l'école à la maison), ne présentaient plus un pluralisme suffisant. Étant engagé dans le Ciane, il a considéré que la possible confusion entre cette population particulière et la source des revendications du Ciane était préjudiciable à la mission de représentation générale des usagers du Ciane. naissance, qui s'étendrait à tous les lieux et à tous les aspects. Elles peuvent associer des parents et des professionnels. La plupart de ces associations ont un fonctionnement différent des précédentes : elles n'ont pas de base territoriale et se sont constituées à partir de groupes de discussion Internet. Comme nous le verrons, ces associations ont développé une forte expertise sur les aspects scientifiques.

On retrouve donc dans le domaine de la périnatalité - et cela, en France comme dans d'autres pays [4] — un échantillon représentatif des différents modèles de mouvements analysés par la littérature [5] : des groupes d'entraide ou self-help groups, des groupes orientés vers la défense des droits et de l'accès aux soins et des groupes appartenant à ce que Brown et al. [6] appellent des embodied health movements, c'est-à-dire des mouvements qui placent l'expérience des individus au cœur de leurs revendications et s'appuient sur la science pour étayer ces revendications.

\section{Associations engagées dans de multiples activités}

Bien que les associations membres du Ciane aient été créées avec des missions assez différentes, en pratique, elles se 
déploient sur un spectre d'activités qui se recoupent d'une association à l'autre. On peut distinguer :

- des activités orientées vers la «communauté » ou les adhérents : permanence téléphonique, échanges, groupes de parole, entraide, information personnalisée, « orientation » vers des professionnels ou des établissements adaptés à la demande... À l'exception de certaines associations très engagées dans l'action politique, ces activités sont partout développées. Elles constituent souvent le cœur de l'association et permettent un renouvellement de ses membres ;

- des activités orientées vers un public plus large : conférences, publications, sites web, expositions. Les associations qui se lancent dans ce type d'activités — et elles sont en nombre significatif — décident de fait d'investir l'espace public pour y faire valoir une certaine conception de ce qui concerne la périnatalité. Elles rentrent dans une démarche plus ouvertement politique ;

- des activités orientées vers les professionnels et les organisations de santé : des associations cherchent à créer un espace d'échanges et de collaboration en vue d'amener « spontanément » certains changements, par exemple en allant à la rencontre des professionnels et des établissements pour prendre connaissance de leur mode de fonctionnement et présenter leur propre point de vue ;

- des activités de représentation dans des instances officielles : ces activités, faibles au moment de la constitution du Ciane, se sont progressivement étoffées, notamment sous l'effet d'une demande extérieure.

Ces quatre types d'activités, dont nous donnerons des exemples concrets dans la suite, représentent plus ou moins une gradation sur un axe qui va de l'individuel au collectif, du privé au public. Certaines associations se positionnent sur les quatre activités, d'autres sur les deux premières, d'autres encore sur les deux ou trois dernières... Ce positionnement est en partie lié à l'origine de l'association : une association créée dans un but de transformation des pratiques va être beaucoup plus investie dans les dernières activités que dans la première, mais le lien est loin d'être mécanique. Notamment, sous l'effet conjugué de la loi de 2002 et de leur appartenance au Ciane, une tendance se dessine vers l'implication des responsables d'associations au départ tournées vers le soutien et le partage dans des activités plus politiques de représentation. On assiste donc à une hybridation croissante des associations qui déploient leur action de l'entraide à la représentation.

\section{Ciane au jour le jour}

Le Ciane a, dès l'origine, fonctionné sur la base d'échanges de mails au travers d'une liste de discussion. Chaque asso- ciation membre doit déléguer au moins un représentant pour suivre et participer aux débats de la liste. En pratique, cette liste compte près de 80 membres. Comme sur toutes les listes de discussion, la participation des différents membres est inégale : sur chaque période d'un an, entre 25 et 40 personnes interviennent effectivement, parmi lesquelles une moitié environ ont une contribution significative — de l'ordre d'un message par semaine en moyenne - sachant que, certaines années, les plus prolixes ont envoyé de 6 à 20 messages par semaine.

D'où viennent les contributeurs les plus actifs ? Si l'on considère les 30 personnes qui ont — depuis la création du Ciane — le plus envoyé de messages, on observe que :

- 12 d'entre elles viennent d'associations locales ; parmi elles, une personne a participé aux échanges de la liste Naissance ;

- 12 autres personnes sont issues d'associations créées à partir des diverses listes de discussion évoquées plus haut;

- quatre personnes appartiennent à la Leche League, dont deux ont participé à la liste Naissance ;

- une personne était en charge de l'association Naissance et Citoyenneté, créée à l'instigation des dossiers de l'obstétrique (la principale revue médicale destinée aux sagesfemmes) et ayant vocation à rassembler usagers et professionnels ;

- enfin, la dernière est issue du Collectif interassociatif sur la santé (CISS).

On constate donc la coexistence de différents mondes qui, jusque-là, semblaient plutôt séparés, celui des associations ancrées territorialement et celui des groupes issus d'Internet, auxquels il faut ajouter celui des grandes associations nationales comme le CISS.

Les différentes catégories de personnes apportent des ressources spécifiques au groupe : les associations locales ont une connaissance fine du terrain, des difficultés que rencontrent les femmes et les couples, de l'organisation du système de soins et de son évolution et, pour certaines, du fonctionnement des instances locales auxquelles les usagers participent. Les groupes issus d'Internet ont développé de très fortes capacités d'analyse et de construction d'argumentaires étayés, ils ont une bonne connaissance de la littérature médicale et se sont dotés d'outils bibliographiques. Autrement dit, la diversité des associations permet de conjoindre les deux formes d'expertise analysées dans la littérature consacrée aux mouvements de patients et d'usagers de la santé : une expertise tirée de l'expérience ou experiential knowledge [7] et une expertise profane qui résulte de l'appropriation par les non-professionnels de connaissances scientifiques et dont les associations sur le sida constituent le cas emblématique [8,9]. Nous présentons dans la suite deux exemples d'associations qui illustrent cette complémentarité des apports. 
Association Bien Naître de Lyon : d'une cause locale à une implication nationale

Cette association est née dans un contexte de fusion de deux maternités à la fin des années 1990. L'une des deux maternités était réputée pour la qualité de son écoute des parents qui allait de pair avec une médicalisation mesurée et négociable entre parents et professionnels. À l'annonce de la fusion, des parents se sont mobilisés, d'abord sous la forme d'un collectif informel, transformé en 2001 en association, pour que ce mode de fonctionnement puisse perdurer dans la nouvelle entité. Cette action n'a pas débouché sur les résultats escomptés : assez rapidement après la fusion effective, les praticiens issus de la maternité défendue ont démissionné et le quart des sages-femmes a quitté l'établissement.

Exactement à la même période, une histoire tout à fait similaire s'est déroulée dans l'agglomération de Dijon ainsi qu'à Nantes ${ }^{3}$. Les trois associations ont alors amorcé une reconversion réussie dans les cas lyonnais et nantais, conduisant à l'étiolement progressif de l'association dans le cas dijonnais. Pour Bien Naître (Lyon), il s'agissait de « promouvoir et valoriser les démarches favorisant le choix pour les parents, en matière d'accompagnement de la grossesse et de la naissance $»^{4}$. À partir de là, l'association s'est engagée dans une série d'activités qui recoupent plus ou moins le spectre décrit précédemment : soutien des parents, relations avec les professionnels, représentation. L'association assure une permanence téléphonique afin d'informer et d'aider les parents, elle organise chaque mois des " cafés-parents ». Pendant près de deux ans, elle a exporté de manière originale le modèle des cafés-rencontres en tenant des cafés «professionnels » qui avaient pour objectif de renforcer le dialogue entre les parents et les professionnels. Elle s'est investie dès 2002 dans la représentation des usagers. Enfin, Bien Naître (Lyon) a aussi très tôt cherché à nouer des relations avec d'autres groupements : une des membres actifs était présente par exemple lors d'un colloque organisé à Paris par une association créée en 2000, Naissance et Citoyenneté, qui avait l'ambition d'être un groupe de pression national associant usagers et professionnels. Dès qu'il a été question de la constitution du Ciane, l'association s'est mobilisée et s'est investie dans la rédaction de la plate-forme de propositions.

\footnotetext{
3 À Nantes, cet épisode a conduit à la réactivation d'une association intitulée elle aussi Bien Naître, qui avait été fondée dans les années 1980 et s'était impliquée dans la création d'une maternité " alternative », soutenue par la mutualité française. Lorsqu'un projet de regroupement des cliniques mutualistes de l'agglomération nantaise voit le jour à la fin des années 1990, l'association se remet en ordre de marche afin de préserver le modèle élaboré une dizaine d'années plus tôt.

${ }^{4}$ Tel qu'énoncé dans la modification d'objet parue au Journal officiel du 15 octobre 2005 .
}

Deux membres de l'association se sont déplacés à Paris lors des états généraux de 2003. C'est l'une d'entre elles qui a été élue présidente du Ciane.

Cette association s'est définie une position particulière dès le départ que l'on pourrait qualifier de réformiste et de généraliste. Elle se donne pour objectif de représenter l'ensemble des usagers dans la diversité de leurs aspirations et d'obtenir, par la négociation, des transformations du système qui permettent de mieux prendre en compte ces aspirations. Elle combine une double orientation vers les usagers et vers les professionnels et apporte au Ciane cette expérience du terrain et des relations au quotidien avec des professionnels. De ce point de vue, le fait que la présidente du Ciane vienne de cette association illustre bien le positionnement du Ciane lui-même.

\section{Alliance francophone pour l'accouchement respecté (Afar), approprier les connaissances médicales}

L'Afar est née sur un coup de tête : une des personnes fondatrices de la liste Naissance suggère d'organiser — sur le modèle de la semaine mondiale de l'allaitement organisée depuis 1997 - une semaine mondiale de l'accouchement respectée (Smar) et de créer une association support pour cet événement. Un mois et demi plus tard, en mai 2003, les statuts sont déposés après qu'ait été défini l'objet de cette association :

«Considérant que les femmes et les couples n'ont plus le choix de l'accouchement ; considérant que c'est devenu un acte hypermédicalisé pour toutes ; considérant que c'est un moment privilégié dans la vie d'une femme, d'un couple, qui de plus génère des droits, l'Afar informe des choix possibles, et agit pour défendre les libertés individuelles. L'Afar n'a ni théorie, ni dogme à diffuser, et est totalement libre et indépendante de toute école ou doctrine médicale, religieuse ou politique. »

L'énoncé même des objectifs et des moyens de l'Afar résulte en grande partie de l'apprentissage collectif dont la liste Naissance a été le siège, du principe d'un choix ouvert pour les parents à l'importance d'une information la plus loyale possible, incluant les aspects médicaux. Les moyens dont se dote l'association sont diversifiés : diffusion d'information (documents, conférences), mobilisation autour d'un événement (Smar), mais aussi positionnement sur le terrain du droit (constitution de partie civile lors de procès), mise en relation entre usagers et professionnels ouverts à des demandes plus variées que celles qu'acceptent les maternités et constitution d'une banque de données sur les dernières informations médicales disponibles. La base bibliographique représente un des résultats les plus aboutis du travail 
de l'Afar, elle comprend plus de 2200 références mises à disposition sur un site public.

Ce travail bibliographique de fond est articulé aux manifestations publiques organisées pour la Smar et qui, chaque année, sont axées sur un thème particulier : l'épisiotomie la première année, puis les positions de l'accouchement, le temps de l'accouchement, la césarienne, etc. Toutes questions qui renvoient à des «nœuds" dans l'organisation de la naissance en France et interrogent les arguments au nom desquels les professionnels imposent des contraintes aux femmes.

L'Afar cherche à la fois :

- à équiper les individus pour qu'ils soient en mesure, face aux protocoles et aux habitudes des professionnels, d'appuyer leurs choix sur une argumentation rigoureuse ;

- à accompagner les personnes qui chercheraient à faire reconnaître leurs droits devant les instances existantes ;

- et à alerter l'opinion publique sur un certain nombre de questions.

Le levier de changement se situe plutôt au niveau individuel, mais c'est en collectivisant et en publicisant les cas et surtout en les insérant dans un macroargumentaire scientifiquement robuste qu'il est susceptible d'opérer.

\section{Comment le Ciane ?}

Nous venons d'insister sur la diversité des associations composant le Ciane, de leur ancrage territorial et sur leurs histoires contrastées : comment à partir de cette mosaïque un collectif a-t-il pu se constituer ? De quelle manière est-on arrivé à la définition d'une plate-forme commune alors que les associations ont des objets spécifiques? Il faut noter que le Ciane n'est pas la première initiative en la matière. Nous avons déjà mentionné une association, Naissance et Citoyenneté, créée en 2000 à l'instigation des Dossiers de l'obstétrique, principale revue médicale destinée aux sages-femmes et dont l'ambition était de constituer un groupe de pression à l'échelle nationale rassemblant professionnels et usagers. Cependant, il existait depuis 1993 une fédération, Naissance et Libertés, qui regroupait une quinzaine d'associations d'usagers réparties aux quatre coins de la France et qui s'était donnée des objectifs assez similaires au Ciane. Pourquoi le Ciane s'est-il imposé là où deux autres tentatives de regroupement avaient échoué ?

La réponse tient en deux points principaux : d'une part, bien que ces différents groupes aient mené des existences assez autonomes les uns vis-à-vis des autres, il existait, souvent grâce aux individus, des liens même tenus qui ont permis que se constituent des références partagées ; d'autre part, le Ciane s'est doté d'outils qui permettent une coordi- nation à distance et pallient ainsi en partie l'éclatement et l'éparpillement géographique.

Nous avons vu dans la présentation de Bien Naître comment les militantes de cette association avaient activement recherché le contact avec d'autres groupes ; de même, la liste Naissance a réussi à attirer, parmi d'autres, deux présidentes de la Leche League, qui ont participé au Ciane, ainsi que la présidente de la fédération Naissance et Libertés. Une des fondatrices de la liste Naissance était animatrice de la Leche League, en contact avec une association membre de la fédération Naissance et Libertés ; elle a été la première présidente de Naissance et Citoyenneté, a créé l'Afar, etc. Même si aucune relation n'était formalisée, les liens tissés par les individus constituaient un réseau serré : des idées, des connaissances, des textes ont pu circuler avec plus ou moins de fluidité d'un groupe à l'autre et former ainsi la trame d'une culture commune. D'autres liens sont passés par des tiers, notamment par une association britannique AIMS (Association for the Improvement of Maternity Services), qui a été " présente » d'une manière ou d'une autre tout au long de cette histoire : ainsi Beverley Beech, sa présidente, participait dès 1993 à la conférence organisée à Toulouse par la toute jeune fédération Naissance et Libertés. En 2000, une des membres de la liste Naissance fait une présentation de l'histoire et de l'action d'AIMS lors d'une autre conférence qui voit la création de Naissance et Citoyenneté. Des relations entre Beverley Beech et certains membres de la liste Naissance se mettent en place, de sorte que lorsque l'Afar est créée, le modèle de militantisme proposé par AIMS sert d'inspiration forte de l'avis d'un de ses créateurs pour lequel « ce qu'avait fait l'AIMS en Angleterre, c'est-à-dire la défense de points de vue forts appuyée sur la mobilisation de connaissances scientifiques et juridiques, était exemplaire ».

Il n'en a pas moins fallu du temps pour arriver à construire une plate-forme de propositions cohérente : la première version, rédigée dans l'urgence en 2003, se présentait comme une liste un peu hétérogène de 40 propositions ; ce n'est qu'après une certaine vie commune ponctuée par un moment fort, les états généraux organisés par le Ciane en 2006, qu'un texte plus synthétique, organisé autour de trois idées fortes - la diversification de l'offre, l'instauration de pratiques plus respectueuses, la mise à disposition d'une information de qualité — a pu être produit [10].

La fédération Naissance et Libertés avait en fait des ambitions assez voisines de celles du Ciane ${ }^{5}$. S'il est difficile de dire a posteriori pourquoi elle a échoué là où le Ciane $\mathrm{a}$ réussi, on peut mettre en évidence deux différences, au-delà

\footnotetext{
$\overline{5}$ Dans les actes de la conférence de 1993 [11], on peut lire cela dans l'introduction : " On peut dès aujourd'hui favoriser des approches de la naissance plus respectueuses des compétences de la mère et de son bébé, de la liberté, de la responsabilité et de l'intimité des parents avec leur enfant (...) et tout aussi sûres que les pratiques actuelles. ».
} 
du contexte plus favorable à la représentation des usagers à partir de 2002 :

- la fédération était constituée d'associations ancrées dans un territoire et pour beaucoup tournées vers les usagers : les associations thématiques comme l'Afar ou Césarine qui sont centrées sur la constitution d'une expertise spécifique n'existaient pas dans les années 1990 ;

- en l'absence d'Internet, la coordination était sans doute plus difficile.

En effet, Internet joue un rôle crucial dans la vie et les échanges du Ciane : la coordination se fait pour l'essentiel par l'intermédiaire des listes de discussion et du site web, qui utilise la technologie du wiki ${ }^{6}$ : sur le site, figure la liste la plus exhaustive possible des actions entreprises par le Ciane ainsi que les comptes rendus des diverses réunions auxquelles participent les membres du Ciane. On y trouve également la copie de tous les courriers, communiqués de presse, présentations dans des conférences, articles produits par les membres du Ciane, de sorte que le site joue le rôle d'une sorte d'archive partagée : certaines discussions — difficiles à reconstituer a posteriori, car elles consistent en une longue série de messages séparés - y sont retranscrites, de manière à permettre un retour synthétique à un moment donné de la discussion et de mettre en réserve, pour une utilisation ultérieure, les arguments élaborés à cette occasion. Enfin, le site sert aussi d'outil de formation pour les personnes qui voudraient s'engager dans la représentation d'usagers.

\section{Conclusion}

Le Ciane agrège des mouvements très divers dans leur histoire, leurs activités et leurs modes d'action : cette diversité, qui demande un vrai travail en interne pour arriver à construire des positions communes, est aussi une richesse, car elle permet de cumuler une pratique du militantisme organisé, une expérience du « terrain », une culture de la discussion argumentée ainsi qu'une maîtrise de la littérature spécialisée.
Il se présente comme un mouvement original entièrement tourné vers l'action politique au sens large : il s'agit de faire pression sur les acteurs concernés, et notamment sur les pouvoirs publics pour transformer l'organisation de la périnatalité. Sollicité pour participer à des groupes d'experts de la HAS, dans les instances des réseaux de périnatalité etc., pour publier des articles ou donner des conférences, le Ciane bataille pour construire et maintenir son expertise qui se situe à l'articulation entre les connaissances scientifiques et médicales et l'expérience des usagers : c'est à cette condition qu'il peut prétendre être entendu des professionnels. Sa composition comme son organisation sont des éléments cruciaux dans ce combat.

\section{Références}

1. Phan E (2009) La remise en cause de pratiques médicales professionnelles de la part des usagers de la périnatalité. Quelles en sont les origines historiques, la légitimité et les moyens aujourd'hui ? Rev Méd Périnat 1(4):207-12

2. Weiner L (1994) Reconstructing motherhood: the La Leche League in postwar America. J Am Hist 80(4):1357-81

3. Sandre-Pereira G (2005) La Leche League : des femmes pour l'allaitement maternel (1956-2004). Clio 21:174-87

4. Goer H (2004) Humanizing birth: a global grassroots movement. Birth 30(4):308-14

5. Rabeharisoa V, Callon M (2000) Les associations de malades et la recherche. I. Des self-help groups aux associations de malades. Med Sci 16:945-9

6. Brown P, Zavestoski S, McCormick S, et al (2004) Embodied health movements: new approaches to social movements in health. Sociol Health Illn 26:50-80

7. Borkman T (1999) Understanding self-help/mutual aid: experiential learning in the commons. Rutgers University Press, New Brunswick and London

8. Epstein S (1995) The construction of lay expertise: AIDS activism and the forging of credibility in the reform of clinical trials, science. Technol Human Values 20:408-37

9. Barbot J (2002) Les malades en mouvements : la médecine et la science à l'épreuve du sida. Balland, Paris

10. Collectif Ciane (2007) Des états généraux de la naissance à la Plate-forme périnatalité. Spirale 41:28-40

11. Collectif (1993) Oser la vie. Actes du forum « Naissances et libertés ». Fédération Naissance et Libertés, Toulouse

\footnotetext{
${ }^{6}$ Cette technologie, mise en œuvre sur Wikipédia, permet le travail collaboratif sur les pages du site.
} 